\title{
EL IMPACTO DE LA PANDEMIA DE COVID 19 EN EL DIAGNÓSTICO DE CÁNCER EN UN SERVICIO DE PATOLOGÍA EN EL SUR DE BRASIL
}

\section{ARTÍCULO ORIGINAL}

KLOCK, Julia Cristhina Monteiro¹, Borges, Giuliano Santos², OGATA, Daniel Cury ${ }^{3}$, KLOCK, Clóvis ${ }^{4}$

KLOCK, Julia Cristhina Monteiro. Et al. El impacto de la pandemia de covid 19 en el diagnóstico de cáncer en un servicio de patología en el sur de Brasil. Revista Científica Multidisciplinar Núcleo do Conhecimento. Año. 06, Ed. 11, Vol. 14, págs. 182-190. Noviembre 2021. ISSN: 2448-0959, Enlace de acceso: https://www.nucleodoconhecimento.com.br/salud/servicio-de-patologia, $\quad$ DOI: 10.32749/nucleodoconhecimento.com.br/salud/servicio-de-patologia

\section{RESUMEN}

La pandemia de Covid-19 ha tenido un impacto significativo en la vida de las personas. Uno de estos impactos se puede verificar en el diagnóstico de otras enfermedades, especialmente el cáncer. Problema: ¿Cuál fue el impacto en el diagnóstico de nuevos casos de cáncer en la región cubierta por un servicio de patología en el sur de Brasil durante la pandemia? Objetivo general: Evaluar si hubo una disminución en el número de diagnósticos de cáncer en un servicio de patología en el sur de Brasil durante la pandemia. Metodología: Analizamos los informes de casos realizados en los Laboratorios del Grupo Infolaudo, durante los primeros meses de la pandemia (abril y mayo de 2020) y comparados con los mismos meses de 2019, con el fin de medir el impacto de la pandemia en el diagnóstico de cáncer en un Servicio de Patología en el sur de Brasil. Resultados: Comparando los

\footnotetext{
${ }^{1}$ Estudiante de Medicina - Universidad de Mogi das Cruzes. ORCID: 0000-0002-0834-193X.

${ }^{2}$ Especialidad en Oncología, Graduación en Medicina. ORCID: 0000-0002-0737-7922.

${ }^{3}$ Doctor en Cirugía, Maestría en Cirugía, Especialidad en Patología, Graduación en Medicina. ORCID 0000-00018819-2155.

${ }^{4}$ Doctorando en Oncología, Especialidad en Patología, Graduación en Medicina. ORCID 0000-0001-8456-0061.
}

RC: 103129

Disponible: https://www.nucleodoconhecimento.com.br/salud/servicio-de-patologia 
períodos de abril y mayo de 2019 con el mismo período de 2020, se observó una disminución del $34,2 \%$ en los procedimientos patológicos. Algunos tipos de cáncer, como colon, esófago, mama y próstata, tuvieron una disminución significativa en los diagnósticos, siendo $19.7 \%$, 45\%, 18.2\% y $37.7 \%$, respectivamente. Conclusión: Con el cierre de muchas clínicas y clínicas en las primeras etapas de la pandemia, hubo una disminución en los diagnósticos de cáncer en los laboratorios encuestados.

Palabras clave: Pandemia, Covid 19, cáncer, patología.

\section{INTRODUCCIÓN}

La Organización Mundial de la Salud (OMS) estima que para 2030 habrá 27 millones de nuevos casos de cáncer, 17 millones de muertes por la enfermedad y 75 millones de personas que viven con cáncer en todo el mundo (INCA, 2020).

El cáncer es la principal causa de muerte en los países desarrollados y en desarrollo (ALLGAR y NEAL, 2005). Sin embargo, ciertos tipos de cáncer tienen una alta probabilidad de curación si se detectan en una etapa temprana y se tratan adecuadamente (AMBUSAIDI y AL-BALUSHI, 2012).

Los retrasos en el diagnóstico del cáncer pueden ocurrir a lo largo del diagnóstico, de acuerdo con los niveles de atención médica: paciente, atención primaria y atención secundaria.Los diagnósticos en enfermedades neoplásicas más avanzadas pueden ocurrir cuando el paciente tarda en reconocer y actuar en relación con los síntomas sospechosos (ASCO, 2020). Esta baja conciencia de los síntomas tempranos del cáncer se considera la razón predominante de una presentación tardía, especialmente cuando los síntomas son de naturaleza atípica (ASCO, 2020). Otra posible barrera puede estar relacionada con la alta demanda de servicios médicos especializados, y eventualmente puede retrasar el diagnóstico, especialmente en los servicios públicos (INCA, 2020). 
En Brasil, según EL INCA, en 2020 se estimaron 309.230 nuevos casos de cáncer, en hombres, el cáncer de próstata fue responsable del 29,2\% de los casos estimados. En mujeres, de los 316.140 nuevos casos previstos, el cáncer de mama correspondió al $29,7 \%$. Estos datos no incluyen el cáncer de piel no melanoma (GREEN et al., 2015).

En un caso, el retraso en el diagnóstico del cáncer puede ocurrir en varios niveles.El paciente puede no reconocer los síntomas sospechosos de cáncer o actuar sobre ellos.Es posible que el médico de atención primaria no reconozca a los pacientes con sospecha de síntomas de cáncer y los investigue adecuadamente o los refiera a tiempo.Los pacientes con sospecha clínica, en atención secundaria, pueden no ser vistos a tiempo o pueden ser referidos a la especialidad equivocada.

Durante la pandemia de coronavirus, las medidas preventivas adoptadas, tales como: distanciamiento social y cuarentena, afectaron el diagnóstico inicial de los pacientes con cáncer. Así, el hacinamiento observado en varios hospitales imposibilitaba el diagnóstico y tratamiento adecuado de estos pacientes.

En este escenario, los datos de la muestra local carecen para verificar si la pandemia realmente afectó a este grupo de pacientes y diagnósticos de cáncer.

En vista de esto, el presente artículo tiene como pregunta guía: ¿Cuál fue el impacto en el diagnóstico de nuevos casos de cáncer en la región cubierta por un servicio de patología en el sur de Brasil durante la pandemia? Con el objetivo general de evaluar si hubo una disminución en el número de diagnósticos de cáncer en un servicio de patología en el sur de Brasil durante la pandemia.

Se analizaron los datos del inicio de la pandemia (abril y mayo) de 2020, comparando con los datos de abril y mayo de 2019, de un Servicio de Patología de la Región Sur de Brasil (Grupo Infolaudo) para reconocer la situación actual y los impactos resultantes del período de lockdown en el diagnóstico de este grupo de individuos. 
Se trata de un estudio epidemiológico transversal y cuantitativo de la muestra local.

\section{MATERIALES Y MÉTODOS}

Se analizaron todos los informes de casos de abril y mayo de 2020 y abril de 2019, realizados en los Laboratorios del Grupo Infolaudo, y se compararon entre sí. Los períodos elegidos se definieron debido al inicio del aislamiento social y las medidas de lockdown. Todos los pacientes incluidos en este estudio fueron tratados en la región de Foz del río Itajaí, Alto Vale, gran Florianópolis y el norte y oeste de Rio Grande do Sul (Erechim y Cruz Alta).

Todos los casos diagnosticados con cáncer se incluyeron en el estudio. Se excluyeron los casos que no tenían confirmación del diagnóstico de cáncer. Los datos recogidos fueron incorporados al programa Microsoft Office Excel® 2010 y los resultados fueron presentados en números absolutos y porcentajes. Se trata de un estudio epidemiológico transversal y cuantitativo de la muestra local.

\section{DISCUSIÓN}

Pueden producirse retrasos en la atención primaria en el reconocimiento, la investigación y la derivación de casos con sospecha de neoplasia maligna (ASCO, 2020).Aunque la atención primaria es el primer punto de contacto para los pacientes que utilizan los servicios nacionales de salud en varios países del mundo, el retraso en el diagnóstico del cáncer sigue siendo un problema continuo a este nivel (JONES et al., 2007).Se espera que los médicos de atención primaria identifiquen a los pacientes con posible enfermedad en una etapa temprana. Sin embargo, el diagnóstico de cáncer es relativamente poco común para el médico de atención primaria, ya que más del $80 \%$ de los pacientes tienen síntomas inespecíficos (HARFORD, 2011).De hecho, la principal preocupación de estos profesionales de la salud es diferenciar la minoría de pacientes que necesitan atención urgente de aquellos que probablemente tienen condiciones autolimitados (KAUFMAN et al., 2020). 
En mayo de 2020, la Sociedad Americana de Oncología Clínica (ASCO) publicó un informe especial que recomienda el aplazamiento de cualquier visita a la clínica y cualquier prueba de detección o diagnóstico de cáncer y procedimientos relacionados con la estadificación si este aplazamiento no representa un riesgo de progresión de la enfermedad o empeoramiento del pronóstico (LONDON et al., 2020).

Algunos estudios internacionales muestran que la disminución en el diagnóstico de cáncer en los primeros meses de la pandemia fue del $65,2 \%$ de los nuevos casos de cáncer (LONDON et al., 2020).

El cribado de algunos tipos de cáncer se vio afectado, con datos que muestran que el cáncer de mama y colon y recto fueron los más afectados con 89,2 y 84,5\% respectivamente (MACLEOD et al., 2009).

En un estudio realizado en Reino Unido, el confinamiento provocó la suspensión de los cribados oncológicos, comprometiendo el diagnóstico precoz de infinidad de pacientes. Solo de esto, los pacientes con cuadro clínico crítico y sintomático fueron dirigidos a la intervención diagnóstica. Los registros de cáncer se utilizaron en el Servicio Nacional de Salud (NHS) a través de una base de datos de hospitales con pacientes de 15 a 84 años, diagnosticados con cáncer de mama (35583), colorrectal (24975) y cáncer de esófago (6744) en 2010 con seguimiento hasta 2014. En pacientes con neoplasia pulmonar primaria (29305), se utilizó 2012 como año de diagnóstico y 2015 como fecha final de seguimiento. A través de un diagrama de flujo para definir las vías de los pacientes con cáncer dentro del NHS, se realizó una estimación para evaluar las consecuencias del retraso diagnóstico en este grupo de pacientes, durante un período de 12 meses, a partir de marzo de 2020 (fecha de lockdown),contextualizando con su impacto 1,3 y 5 años después del diagnóstico inicial. En esta metodología se consideraron tres vías o flujos de estos pacientes, correspondientes del mejor al peor escenario. En base a esto, se estimó el impacto real de la supervivencia a los 1,3 y 5 años después del diagnóstico, calculando así el número total de muertes atribuidas por cáncer y el número total de años de vida perdidos, en comparación con los datos previos a la pandemia. 
En todos los escenarios, se estimó un aumento del 7,9\% al 9,6\% de las muertes por cáncer de mama dentro de los 5 años posteriores al diagnóstico, lo que significa de 281 a 344 muertes más, respectivamente. En el cáncer colorrectal, el aumento fue de $15,3 \%$ (1445) a 16,6\% (1563) y en los cánceres de pulmón, este aumento fue de $4,8 \%$ (1235) a 5,3\% (1372). Finalmente, el aumento observado en pacientes con cáncer de esófago fue de 5,8\% (330) a 6\% (342). Estos datos muestran que ha habido un aumento significativo en las muertes prevenibles en el Reino Unido, probablemente debido a medidas restrictivas y de aislamiento social (MARINGE et al., 2020).

Otro estudio publicado por Kaufman et al. (2020) observó una reducción del 40\% en la incidencia semanal de cáncer en los Países Bajos y del 75\% en el Reino Unido desde el comienzo de la pandemia de covid-19. Este estudio utilizó una metodología similar a la nuestra, evaluando los registros de una base de datos de enero a abril de 2019 , en comparación con el mismo período de 2020. Notaron caídas significativas en el diagnóstico de neoplasias malignas, in situ y comportamiento indeterminado. Durante la pandemia, el número semanal de casos se redujo en más de un $46 \%$ en los seis tipos de cáncer más comunes, con variaciones del $24,7 \%$ en los casos de neoplasia pancreática y del $51,8 \%$ en los casos de mama (OMS, 2016).

Otro artículo, publicado por investigadores del Sidney Kimmel Center en Filadelfia y el NHS, mostró una reducción del $89,2 \%$ en la detección del cáncer de mama y una disminución del $85,5 \%$ en las colonoscopias, que es una herramienta importante para el diagnóstico temprano del cáncer colorrectal (VOSE, 2020).

Todos los casos del Grupo Infolaudo en los períodos de marzo y abril de 2020 se compararon con el período de marzo y abril de 2019. Se seleccionaron los meses de marzo y abril, ya que fueron los primeros meses de lockdown.

En 2019, en abril y mayo, los Laboratorios del Grupo Infolaudo recibieron 5524 procedimientos patológicos, de los cuales 4634 fueron lesiones benignas y 890 lesiones malignas, lo que representa el 16,1\%. En 2020, en el mismo periodo, se presentaron 3635 procedimientos, con 2.875 lesiones benignas y 760 lesiones 
malignas, lo que representa un 20,9\% maligno. Hubo una disminución del 34,2\% en los procedimientos patológicos. (Gráfico 1)

Gráfico 1: Disminución de los procedimientos patológicos en abril y mayo de 2020 en relación con el mismo período de 2019.

\section{Lesões benignas X Lesões malignas}

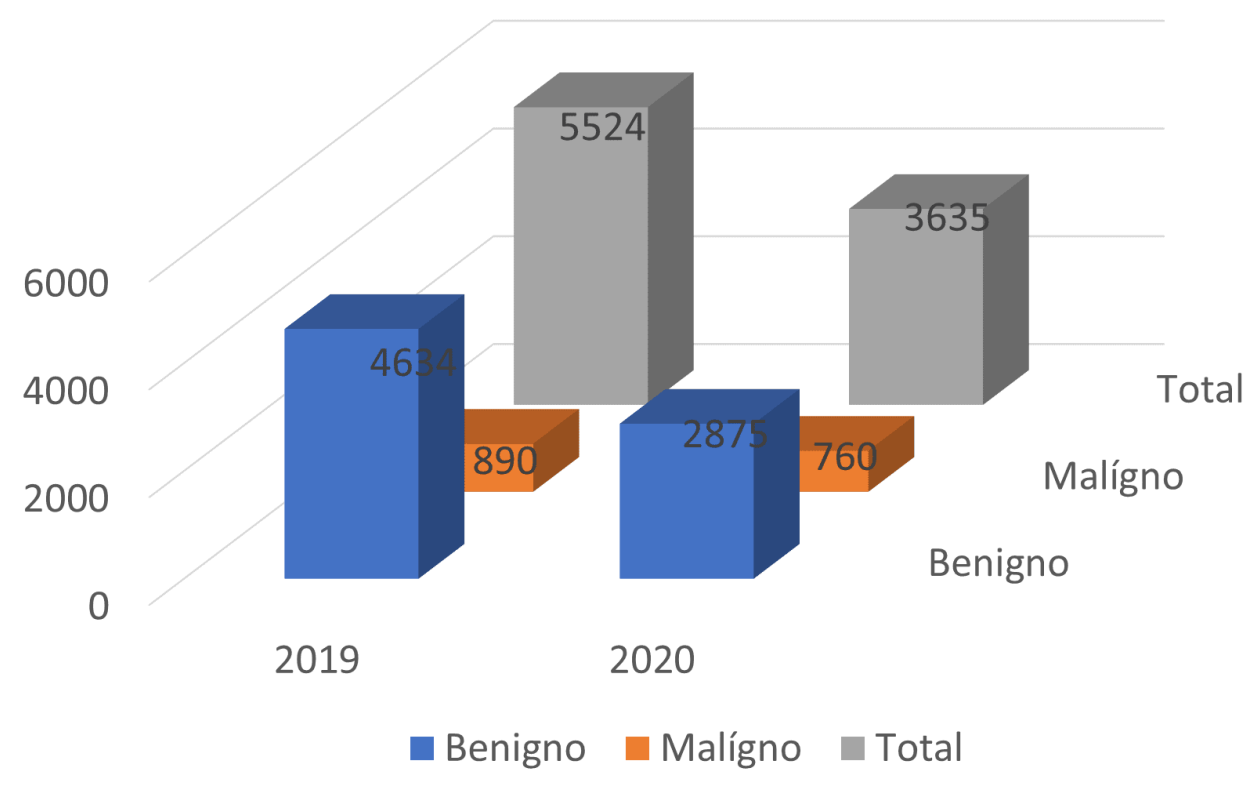

Fuente: autores

En general, en todos los diferentes procedimientos, hubo una disminución en el diagnóstico, ya sean biopsias o muestras quirúrgicas. Algunos tipos de procedimientos tuvieron una disminución más marcada, como las cirugías electivas mayores que requirieron la hospitalización del paciente.

Algunos tipos de cáncer (Gráfico 2) tuvieron una disminución más significativa, como el colon (19,7\%), el esófago (45\%), el de mama $(18,2 \%)$ y la próstata $(37,7 \%)$.

Gráfico 2: disminución de los diagnósticos de cáncer (por órganos) más afectados en abril y mayo de 2020 en comparación con el mismo período de 2019. 


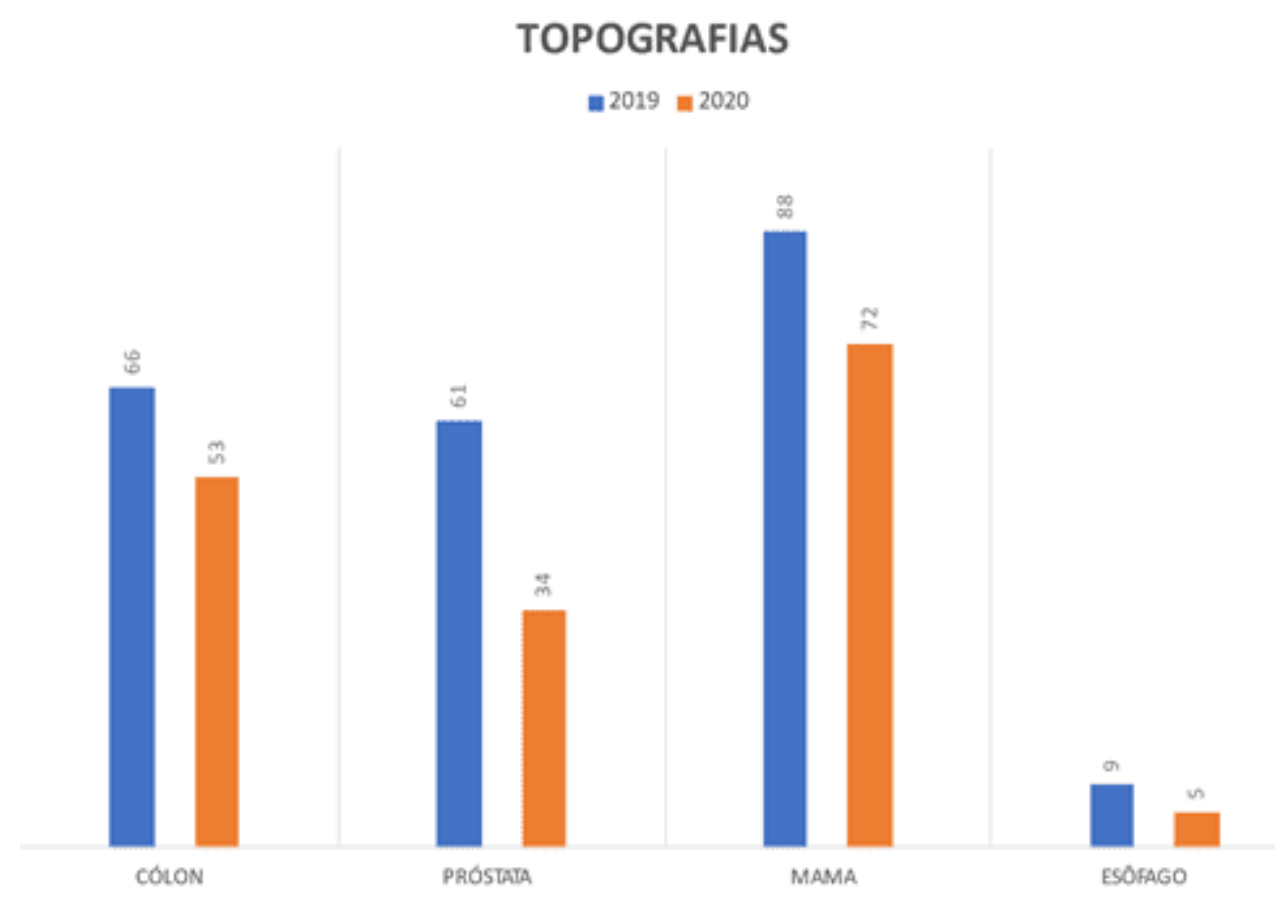

Fuente: autores.

La principal disminución fue en los casos que requerirían algún procedimiento invasivo, y en los casos de sedación del paciente. Otros tipos, como los cánceres de piel, no disminuyeron en el mismo período.

Los bloques quirúrgicos de la mayoría de los hospitales también limitaron las cirugías electivas, debido a la necesidad de dejar camas vacías, especialmente camas de UCl.

Con el impacto del cierre de muchas clínicas y clínicas en las primeras etapas de la pandemia, hubo un gran impacto en estos diagnósticos. Además del miedo a que los pacientes salieran de sus casas, que también fue uno de los factores que ayudaron en esta disminución.

Este estudio tiene algunas limitaciones. En primer lugar, solo se evaluó el impacto del bloqueo en el diagnóstico en un grupo de laboratorios, aunque se trata de un servicio grande. Los resultados del presente estudio, por lo tanto, no son necesariamente aplicables a otros centros, por lo que serían necesarios estudios 
multicéntricos para validar estos resultados a escala nacional. Es posible que esto indujesa un sesgo de selección. Sin embargo, este método de selección de pacientes fue idéntico para todos los períodos estudiados, lo que permitió la comparabilidad.

\section{CONSIDERACIONES FINALES}

Volviendo a la pregunta correcta: ¿Cuál fue el impacto en el diagnóstico de nuevos casos de cáncer en la región cubierta por un servicio de patología en el sur de Brasil durante la pandemia? A través del estudio presentado, se pudo concluir que los datos analizados en los informes de pacientes atendidos en el Grupo Infolaudo mostraron que el diagnóstico de algunos tipos de cáncer disminuyó en el periodo inicial de la pandemia en 2020 con el periodo analizado de 2019, mostrando que la pandemia afectó a este tipo de diagnósticos. También hubo una gran disminución en el número de biopsias y muestras quirúrgicas recibidas en el período. Esta explicación puede ser multifactorial, y puede estar influenciada por el cierre de servicios de diagnóstico como clínicas y sectores de hospitales, así como por el miedo a los pacientes que buscan atención médica.

\section{REFERENCIAS}

ALLGAR, V. L.; NEAL, R. D. Delays in the diagnosis of six cancers: analysis of data from the National Survey of NHS Patients: Cancer. Br J Cancer, 2005.

AMBUSAIDI, A.; AL-BALUSHI, S. Educação em Saúde no Sultanato de Omã. In: Taylor, N. et al. (ed.). Educação em Saúde em Contexto. Editores Sense. 2012.

ASCO. American Society of Clinical Oncology. COVID-19 Patient Care Information. 2020. Disponível em: https://www.asco.org/asco-coronavirus-information/careindividuals-cancer-during-covid-19. Acesso em: 26 de jun. de 2020.

GREEN, T. et al. Cancer detection in primary care: insights from general practitioners. Ir. J Cancer, 2015. 
HARFORD, J. B. Breast-cancer early detection in low-income and middleincome countries: do what you can versus one size fits all. Lancet Oncol, 2011.

INCA. Instituto Nacional de Câncer. Estimativa 2020: incidência de câncer no Brasil. Rio de Janeiro: INCA, 2020.

JONES, R. et al. Alarm symptoms in early diagnosis of cancer in primary care: cohort study using General Practice Research Database. BMJ, 2007.

KAUfMAN, H. W. et al. Changes in the Number of US Patients With Newly Identified Cancer Before and During the Coronavirus Disease 2019 (COVID-19) Pandemic. JAMA Netw Open, 2020.

LONDON, J. W. et al. Effects of the COVID-19 pandemic on cancer-related patient encounters. JCO Clinical Cancer Inform, 2020.

MACLEOD, $U$. et al. Risk factors for delayed presentation and referral of symptomatic cancer: evidence for common cancers. Br J Cancer, 2009.

MARINGE, C. et al. The impact of the COVID-19 pandemic on cancer deaths due to delays in diagnosis in England, UK: a national, population-based, modelling study. Lancet Oncol, 2020.

VOSE, J. M. Delay in Cancer Screening and Diagnosis During the COVID-19 Pandemic: What Is the Cost? Oncology (Williston Park), 2020.

WHO. World Health Organization. Facts about cancer. 2016. Disponível em: www.who.int

Publicado: Noviembre de 2021.

Aprobado: Noviembre de 2021. 